DOI: 10.1002/ ((please add manuscript number))

Article type:

\title{
On the Role of Contact Resistance and Electrode Modification in Organic Electrochemical Transistors
}

Alexandra F. Paterson, Hendrik Faber, Achilleas Savva, Georgios Nikiforidis, Murali Gedda Tania C. Hidalgo, Xingxing Chen, Iain McCulloch, Thomas D. Anthopoulos, Sahika Inal*

Dr. A. F. Paterson, Dr. A. Savva, Dr. G. Nikiforidis, T. C. Hidalgo, and Prof. S. Inal

Biological and Environmental Science and Engineering Division

King Abdullah University of Science and Technology

Thuwal 23955-6900, Saudi Arabia

E-mail: sahika.inal@kaust.edu.sa

Dr. H. Faber, Dr. M. Gedda, and Prof. T. D. Anthopoulos

Division of Physical Sciences and Engineering

King Abdullah University of Science and Technology

Thuwal 23955-6900, Saudi Arabia

X. Chen and Prof. I. McCulloch

Division of Physical Sciences and Engineering

King Abdullah University of Science and Technology

Thuwal 23955-6900, Saudi Arabia

Keywords: organic electrochemical transistors; contact resistance; electrode modification; morphology/organic semiconductors; self-assembled monolayers 


\begin{abstract}
Contact resistance is renowned for its unfavourable impact on transistor performance. Despite its notoriety, the nature of contact resistance in organic electrochemical transistors (OECTs) remains unclear. Here, by investigating the role of contact resistance in n-type OECTs for the first time, we also report the first demonstration of source/drain electrode surface modification for achieving state of the art n-type OECTs. Thiol-based self-assembled monolayers (SAMs) 4-methylbenzenethiol (MBT) and pentafluorobenzenethiol (PFBT) are used to investigate contact resistance in n-type accumulation mode OECTs made from the hydrophilic copolymer NDI-T2, where the deliberate functionalisation of the gold source/drain electrodes decreases and increases the energetic mismatch at the electrode/semiconductor interface. Although MBT treatment is found to increase the transconductance three-fold, contact resistance is not found to be the dominant factor governing OECT performance. Additional morphology and surface energy investigations show that increased performance comes from SAM-enhanced source/drain electrode surface energy, which improves wetting, semiconductor/metal interface quality and semiconductor morphology at the electrode and channel. Overall, we investigate contact resistance in n-type OECTs for the first time, whilst identifying source/drain electrode treatment as a useful device engineering strategy for achieving state of the art n-type OECTs.
\end{abstract}

\title{
Main Text
}

Contact resistance $\left(\mathrm{R}_{\mathrm{C}}\right)$ has long been established as a performance-limiting factor for many types of transistors. ${ }^{[1] ~[2] ~[3] ~} \mathrm{R}_{\mathrm{C}}$ primarily emerges from a Schottky barrier at the electrode/semiconductor interface, caused by an energetic difference between the work function (WF) and transport level (highest occupied molecular orbital (HOMO)/lowest unoccupied molecular orbital (LUMO)) of the electrode and semiconductor, respectively. The presence of a potential barrier at this critical interface is well-known to inhibit charge carrier injection/extraction, ${ }^{[4]}{ }^{[5]}$ and is particularly problematic in transistors made using wide-bandgap, organic semiconductors (OSCs). ${ }^{[6] ~[7]}$ One such organic-based transistor is the organic field-effect transistor (OFET), namely the organic 
thin-film transistor (OTFT). The magnitude of Rc in OTFTs is determined by a complex interplay between OSC morphology ${ }^{[8]}$ and dielectric material, ${ }^{[9]}{ }^{[10]}{ }^{[11]}$ as well as the size of the Schottky barrier at the OSC/metal interface. ${ }^{[12][13][14]}$ Moreover, $\mathrm{R}_{\mathrm{C}}$ is renowned for limiting OTFT physical dimensions, charge carrier mobilities and accurate parameter extraction. ${ }^{[15]}{ }^{[16]}[17]$

Another type of organic transistor, the organic electrochemical transistor (OECT), has recently established itself as a useful tool for emergent bioelectronic applications, brain and body implants, sensors, signal recorders and neuromorphic systems. ${ }^{[18]}{ }^{[19]}{ }^{[20]}[21]{ }^{[22]}$ OECTs are governed by mixed ionic- and electronic-conductivity, with high transconductance $\left(\mathrm{g}_{\mathrm{m}}\right)$ being an essential OECT parameter, characteristic of efficient ionic-to-electronic signal conversion. ${ }^{[23]}$ Similar to OTFTs, the electronic function of an OECT requires charge carriers to be injected/extracted between an OSC and metal source/drain electrodes. The latter are often made from biocompatible gold ( $\mathrm{Au})$, with a WF $\sim-5 \mathrm{eV} \cdot{ }^{[24]}$ For some OECTs, especially n-types, this WF value results in large energetic mismatches at the Au/OSC interface. ${ }^{[25][26]}{ }^{[27]}$ Yet despite the apparent Schottky barrier, as well as the debilitating impact it can have on transistors, there is very little discussion about $\mathrm{R}_{\mathrm{C}}$ in OECTs. In fact, $\mathrm{R}_{\mathrm{C}}$ is only discussed in two papers with conflicting findings. ${ }^{[28][29]}$ On one hand, Kaphle et al. find $\mathrm{RC}_{\mathrm{C}}$ influences to be so pronounced that it causes a 'bell-shaped' gate voltage $\left(\mathrm{V}_{\mathrm{G}}\right)$ dependent $\mathrm{g}_{\mathrm{m} \cdot}{ }^{[28]}$ On the other hand, Friedlein et al. show $\mathrm{R}_{\mathrm{C}}$ is $\approx 20 \%$ of the total resistance in depletion mode OECTs, reducing $\mathrm{g}_{\mathrm{m}}$ by $10 \%$ at $\mathrm{V}_{\mathrm{G}}<0.2 \mathrm{~V}$, whilst contributing negligibly to the total resistance in accumulation mode OECTs. ${ }^{[29]}$ Furthermore, these studies use p-type OSCs, which typically have deeper HOMO levels compared to the LUMO levels of their n-type counterparts. $\mathrm{R}_{C}$ has not yet been studied in n-type OECTs or investigated independently from the non-monotonic $\mathrm{g}_{\mathrm{m}}$. 
Here, we investigate the impact of $R_{c}$ on solution-processed, accumulation mode n-type OECTs for the first time, by deliberately modifying the energetic mismatch at the metal/OSC interface. The self-assembled monolayers (SAMs), 4-methylbenzenethiol (MBT) and pentafluorobenzenethiol (PFBT), were used to shift the Au WF closer to and further away from the vacuum level, respectively, giving Au, Au:MBT and Au:PFBT source/drain electrodes with $\mathrm{R}_{\mathrm{C} \_A u: M B T}<\mathrm{R}_{\mathrm{C} \_A u}<\mathrm{R}_{\mathrm{C} \_ \text {Au:PFBT. }}$ The MBT-treated OECT performance was found to be greater than current state of the art n-type OECTs. ${ }^{[30]}$ However, further analysis shows $\mathrm{R}_{\mathrm{C}}$ alone is not enough to explain higher performance in MBT-treated OECTs compared to untreated and PFBT-treated devices. Further surface energy characterisation reveals that MBT-treatment preferentially adjusts the surface energy of the Au source/drain electrodes, improving solution-processed polymer wetting and quality of the metal/OSC interface. The latter results in stark morphological differences and is the first demonstration of source/drain electrode SAM treatment for improving OECT mobility, capacitance and transconductance. Overall, the findings in this study are two-fold: (i) we find Rc to be influential - but not dominant - in OECTs, whilst identifying two, distinct Rc-operational regimes at low and high $\mathrm{V}_{\mathrm{G}}$; (ii) we report, for the first time, metal/OSC interface modification as a device engineering strategy for producing state of the art n-type OECTs.

To investigate the impact of $R_{C}$, we identified an n-type OECT system with a pre-existing energetic mismatch between the Au electrodes and OSC transport level. The conjugated polymer poly(N,N'-bis(7-glycol)-naphthalene-1,4,5,8-bis(dicarboximide)-co-2,2'-bithiophene-co-N,N'bis(2-octyldodecyl)-naphthalene-1,4,5,8-bis(dicarboximide) (P-90) (Figure 1a), a naphthalene1,4,5,8-tetracarboxylic diimide (NDI) and bithiophene (T2) copolymer with ethylene glycol side chains, ${ }^{[27]}{ }^{[31]}$ has recently been shown as a relevant and effective OSC for OECTs and their applications. We measured the P-90 LUMO level at $\approx-3.9 \mathrm{eV}$ using ultraviolet photoelectron 
spectroscopy (UPS), giving an approximate mismatch of $\approx 1.1 \mathrm{eV}$ at the Au:P-90 interface. The latter makes this an ideal system to study $\mathrm{R}_{\mathrm{C}}$.

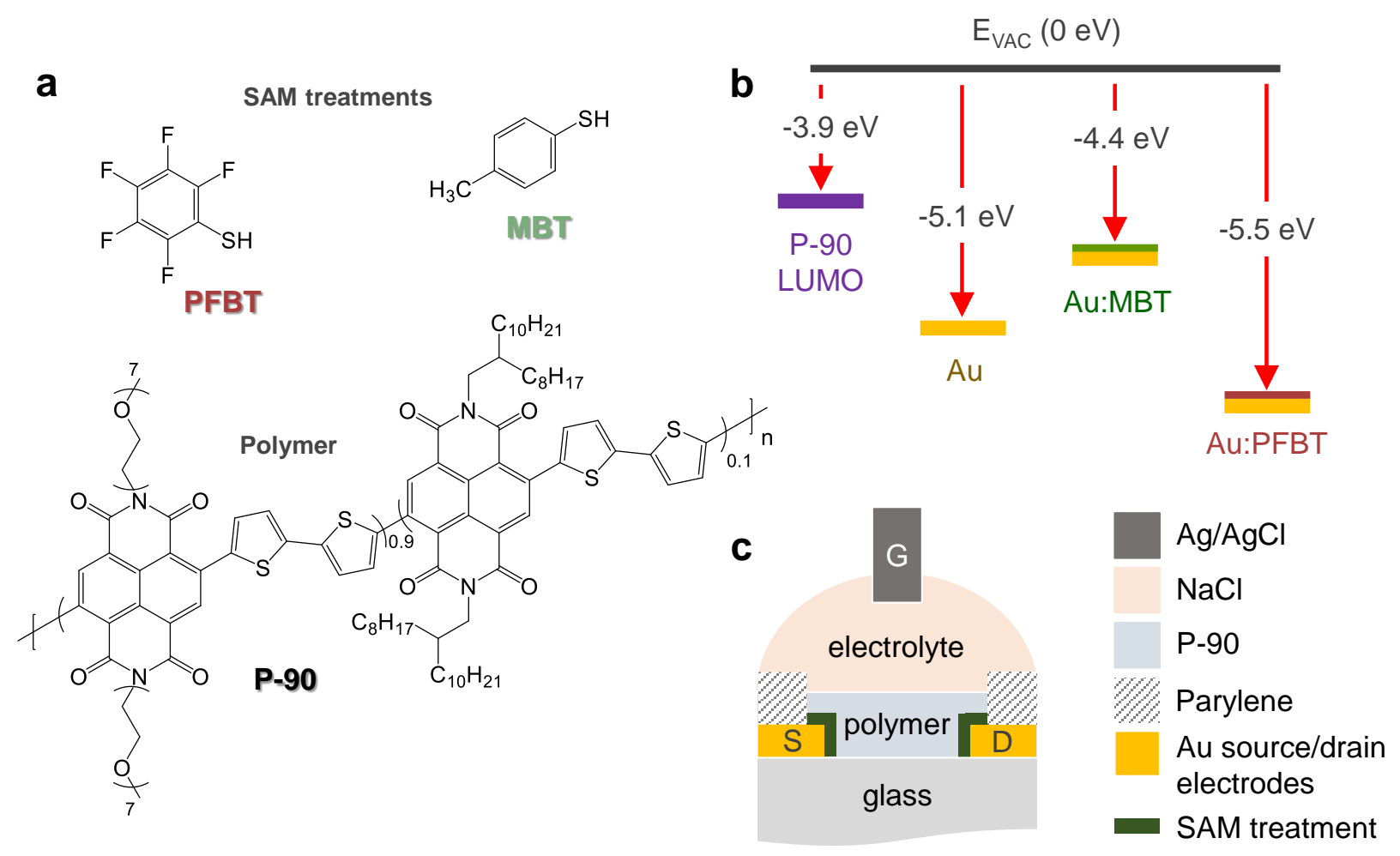

Figure 1. (a) Chemical structure for n-type organic semiconductor naphthalene-1,4,5,8tetracarboxylic diimide-bithiophene with glycol side chains (P-90) and the two self-assembled monolayers, 4-methylbenzenethiol (MBT) and pentafluorobenzenethiol (PFBT), used to modify the surface of the gold source/drain electrodes. (b) Schematic showing the energetic mismatch between the work function (WF) of untreated and treated gold source/drain electrodes and the lowest unoccupied molecular orbital (LUMO) of P-90. The WF of untreated/treated gold is measured using Kelvin Probe, whilst the LUMO of P-90 is measured using UPS. (c) Organic electrochemical transistor (OECT) configuration used throughout this work.

Next, we identified a suitable approach for defining $R_{c}$, by varying the energetic mismatch between the P-90 and Au source/drain (S/D) electrodes. SAMs are widely used to modify the surface potential metal electrodes and improve $R_{C}$ by vacuum alignment. ${ }^{[5]}{ }^{[32]}{ }^{[33]}$ Although the theoretical impact of such approaches are eventually limited physically by surface effects such as the formation of extra interfacial dipoles, ${ }^{[34]}$ or Fermi level pinning at the polaron level, ${ }^{[5]}$ this 
technique is widely employed in both p-type and n-type OTFTs. ${ }^{[35] ~[36] ~[37] ~ I n ~ p a r t i c u l a r, ~ t h i o l-b a s e d ~}$ SAMs produce a monolayer of interfacial electric dipoles on $\mathrm{Au}$ surfaces via gold-sulphur interactions, ${ }^{[32]}{ }^{[38]}$ changing the WF by redistributing the charge density at the Au surface. ${ }^{[39] ~[32] ~}$ Whether the WF is increased or reduced is highly dependent on the SAM character; namely the dipole moment and electron withdrawing/donating nature of the SAM tail, and the chemical nature of the anchoring head group. ${ }^{[40]}{ }^{[41]}{ }^{[42]}$ Therefore we employed two types of thiol-based SAMs, 4-methylbenzenethiol (MBT) ${ }^{[43]}{ }^{[44]}$ and pentafluorobenzenethiol (PFBT) (Figure 1a), ${ }^{[33]}$ to reduce and increase, respectively, the energetic mismatch between Au and P-90. The WF of the untreated and functionalised electrodes were measured using Kelvin Probe (KP), finding the energetic mismatch in the Au, Au:MBT and Au:PFBT P-90 OECTs to be 1.2, 0.5 and $1.6 \mathrm{eV}$, respectively (Figure 1b). We hypothesise that, if $\mathrm{R}_{\mathrm{C}}$ indeed influences OECTs, the overall performance of P-90 OECTs should be improved by MBT and worsened by PFBT.

Having identified appropriate systems, we investigated the impact of the $\mathrm{Au}, \mathrm{Au}: \mathrm{MBT}$ and Au:PFBT S/D electrodes on P-90 OECT performance. OECTs (Figure 1c) were made with channel length $10 \mu \mathrm{m}$ and channel width $100 \mu \mathrm{m}$, and gated with an $\mathrm{Ag} / \mathrm{AgCl}$ electrode immersed in $0.1 \mathrm{M} \mathrm{NaCl}_{(\mathrm{aq} .)}$. All devices were tested under the same positive-bias operating conditions, electrochemically doping P-90 by injecting cations from the electrolyte to the channel. Figure 2a-b show the maximum and average transconductance $\left(g_{m}\right)$ values of the different systems. Compared to the pristine Au:P-90 devices, the peak $\mathrm{g}_{\mathrm{m}}$ taken at $\mathrm{V}_{\mathrm{G}}=0.5 \mathrm{~V}$ is found to be approximately $3 \times$ greater in the best-performing Au:MBT:P-90 devices $\left(\mathrm{g}_{\mathrm{m} \_\mathrm{MBT}}=2.4 \mu \mathrm{S}\right.$ vs. $\left.\mathrm{g}_{\mathrm{m} \_\max }=0.8 \mu \mathrm{S}\right)$ and slightly reduced in Au:PFBT:P-90 devices $\left(\mathrm{g}_{\mathrm{m} \_\max }=0.5 \mu \mathrm{S}\right)$. This trend is further confirmed with statistical variation taken over 6 OECTs, shown in Figure 2b, albeit greater performance variability for Au:MBT:P-90. We note here that current state of the art n-type 
poly(benzimidazobenzophenanthroline) (BBL) OECTs have 2.5× greater $\mathrm{g}_{\mathrm{m}}$ than P-90 for comparable device geometry. [30] [26] [25] Our best-performing SAM-treated OECTs therefore achieve state of the art performance without the need for extra chemical synthesis.
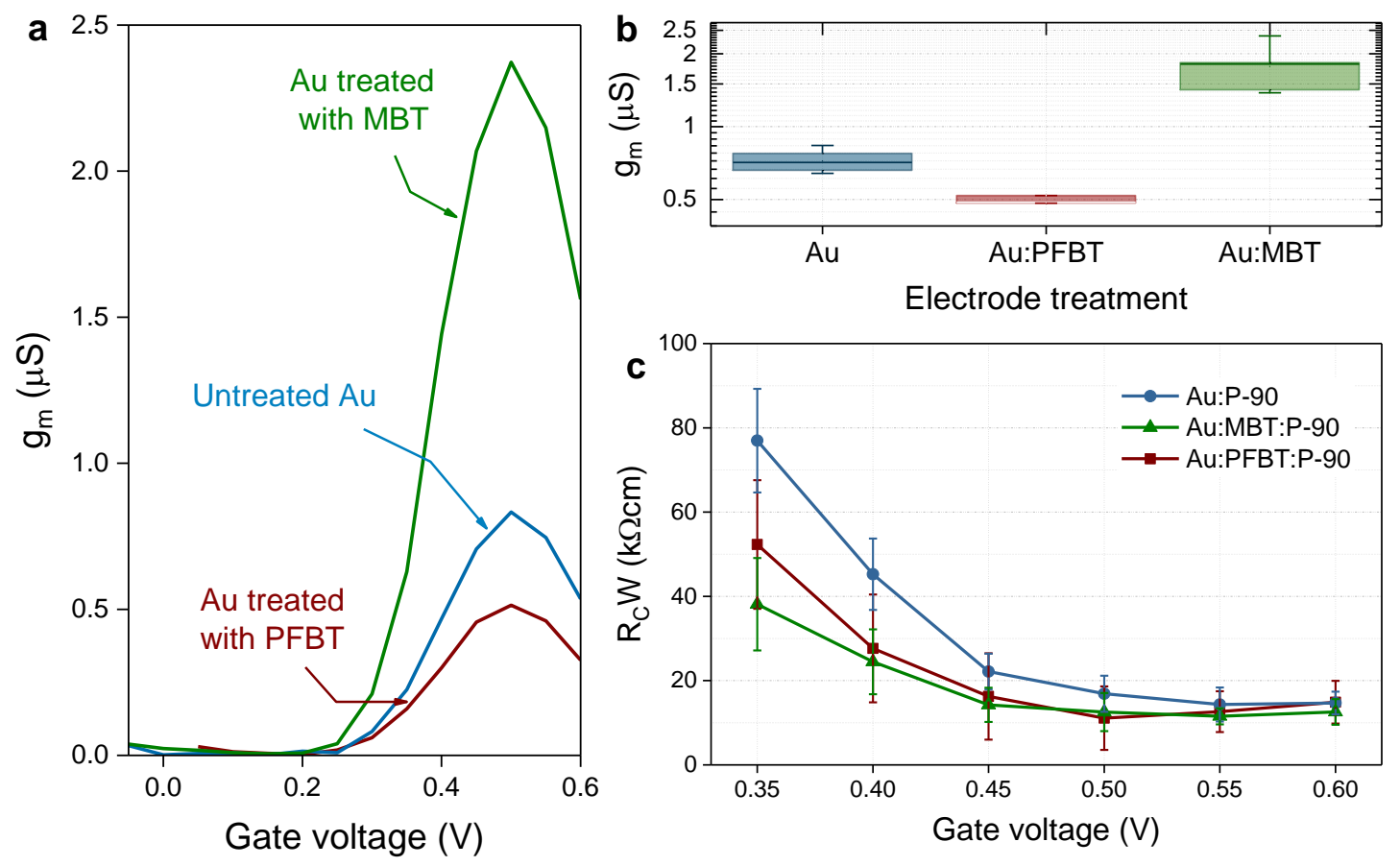

Figure 2. (a) Best-performing organic electrochemical transistors: untreated gold (blue line), gold functionalised with MBT (green line) and gold functionalised with PFBT (red line) measured at $V_{D}=0.6 \mathrm{~V}$. (b) Statistical variation in the maximum transconductance, where $\mathrm{g}_{\mathrm{m}}$ is taken over six organic electrochemical transistors for each system, at $V_{G}=0.5 \mathrm{~V}$. The P-90 thin-films have comparable thicknesses $(\approx 135 \mathrm{~nm})$ in all channels. (c) Summary and comparison of contact resistance $\left(\mathrm{R}_{\mathrm{C}}\right)$ dependency on gate voltage for the Au:MBT:P-90, Au:PFBT:P-90 and Au:P-90 systems, measured at $\mathrm{V}_{\mathrm{D}}=0.6 \mathrm{~V}$, and calculated using the transmission line method.

To investigate whether $\mathrm{R}_{\mathrm{C}}$ is indeed responsible for the observed changes in OECT performance, we used the transmission line method (TLM) to deconvolute $\mathrm{R}_{\mathrm{C}}$ and channel resistance $\left(\mathrm{R}_{\mathrm{CH}}\right){ }^{[45]}{ }^{[46]}{ }^{[47]} \mathrm{R}_{\mathrm{C}}$ was extracted from the output characteristics of OECTs with channel lengths varying from $10 \mu \mathrm{m}$ to $100 \mu \mathrm{m}$, at a fixed width of $100 \mu \mathrm{m}$ (Figure S1). The results in Figure 2c show that $R_{C}$ is highly dependent on $V_{G}$. This variation of $R_{C}$ with $V_{G}$ is typical in OTFTs ${ }^{[15]}[48][8]$ and often associated with a larger/more resistive Schottky barrier at low $\mathrm{V}_{\mathrm{G}}$, 
which reduces in width and height as $\mathrm{V}_{\mathrm{G}}$ is increased. In the presence of a potential barrier, charges can be transported across the depletion region via thermionic emission over the potential barrier, and/or quantum mechanical tunneling across it. In this case, charge carriers tunnel across the depletion region, provided that the latter is thin enough. ${ }^{[49]}[6]$ Following this, Figure 2c suggests two $V_{G}$-dependent $R_{C}$-regimes exhibiting different carrier injection behaviours: (i) a low $V_{G}$ region, where $\mathrm{R}_{\mathrm{C} \_A u: M B T}<\mathrm{R}_{\mathrm{C} \_A u}$ :PFBT $<\mathrm{R}_{\mathrm{C} \_\mathrm{Au}}$, and Au:MBT:P-90 OECTs exhibit superior electron injection/extraction; (ii) a high $\mathrm{V}_{\mathrm{G}}$ region, where $\mathrm{R}_{\mathrm{C} \_ \text {Au:MBT }} \approx \mathrm{R}_{\mathrm{C} \_ \text {Au:PFBT }} \approx \mathrm{R}_{\mathrm{C}_{-} A \mathrm{Au}}$, and all systems have equal injection/extraction properties. The fact that $\mathrm{R}_{\mathrm{C}}$ is greater and more varied at lower $\mathrm{V}_{\mathrm{G}}$, whilst tending to the same values at high $\mathrm{V}_{\mathrm{G}}$, is further substantiated by taking into account errors commonly associated with the TLM method.

If condition (i) is valid, certain behaviors are expected in Au:MBT:P-90 OECTs at low $\mathrm{V}_{\mathrm{G}}$. For instance, we expect a more rapid increase of the channel current ( $\left.\mathrm{I}_{\mathrm{D}}\right)$ with increasing $\mathrm{V}_{\mathrm{G}}$, i.e. higher $\mathrm{g}_{\mathrm{m}}$, which would also translate to a smaller threshold voltage $\left(\mathrm{V}_{\mathrm{T}}\right)$. The latter relationship between $V_{T}$ and $R_{C}$ arises from the $V_{G}$ dependent $R_{C}$ in organic transistors. ${ }^{[8]}{ }^{[15] ~[48]}$ Furthermore, the output curves should produce comparatively higher $\mathrm{I}_{\mathrm{D}}$ at low drain voltage $\left(\mathrm{V}_{\mathrm{D}}\right)$. Indeed, analysis of the OECT data in Figure 2a-b and Figure S2 reveals the different behaviours. Figure 2a shows a sharper increase in $g_{m}$ at $0.25 \mathrm{~V}$, while Figure $\mathbf{S 2 a}$ demonstrates a slight reduction in $\mathrm{V}_{\mathrm{T}}$, with $\mathrm{V}_{\mathrm{T}_{-} \mathrm{MBT}}=0.27 \mathrm{~V}$ and $\mathrm{V}_{\mathrm{T}_{-} A u}=\mathrm{V}_{\mathrm{T}_{-} \mathrm{PFBT}}=0.29 \mathrm{~V}$. Moreover, Figure S2b-d show a significant increase in $I_{D}$ at low $V_{D}$. We note here that gate leakage $\left(I_{G}\right)$ does not influence our analysis (Figure S3). Further evidence of enhanced electron transfer in Au:MBT:P-90 was found using cyclic voltammetry (CV) measurements shown in Figure S4. A qualitative indication of electron transfer efficiency can be gauged from the sharpness and height of the oxidation and reduction peaks, which are significantly improved in Au:MBT:P-90 OECTs. This can be verified 
quantitatively by extracting the standard electron transfer rate constant, КEт, using the Laviron model. ${ }^{[50]}$ Figure S5b-d show linear relationships between the scan rates and measured current in each system, indicative of surface-bound redox processes where Laviron theory can be applied. The resultant KET values were found to be 0.09, 0.12 and $0.06 \mathrm{~s}^{-1}$ for Au:P-90, Au:MBT:P-90 and Au:PFBT:P-90, respectively. The higher KET value suggests MBT increases the ability of transfer reactions to occur at the microelectrode surface. ${ }^{[51]}{ }^{[52]}$ These results further highlight the benefits of SAM-treated S/D electrodes in OECTs.

Point (ii) suggests the initial $\mathrm{R}_{\mathrm{C}}$ differences are compensated for as $\mathrm{V}_{\mathrm{G}} /$ channel conductivity increases. One explanation for $\mathrm{R}_{\mathrm{C}}$-compensation in OECTs is ionic doping. ${ }^{[19]} \mathrm{In}$ brief, as $V_{G}$ is applied and electrons are injected from the Au S/D electrodes, cations are drawn from the electrolyte into the n-type OSC. ${ }^{[19]}$ Electrons are subsequently transferred/donated from the ions to dope the OSC, shifting the Fermi level ${ }^{[53]}$ and reducing the height/width of the Schottky barrier, ${ }^{[54]}$ hence reducing $\mathrm{R}_{\mathrm{C}}{ }^{[55]}$ This results in similar $\mathrm{R}_{\mathrm{C}}$ at high $\mathrm{V}_{\mathrm{G}}$, regardless of the initial $\mathrm{R}_{\mathrm{C}}$ magnitude. We also note that Rc-compensation efficiency may increase as the polymer channel swells with $\mathrm{V}_{\mathrm{G} .}{ }^{[56]}{ }^{[57]}$ Overall, if $\mathrm{R}_{\mathrm{C}}$ is the dominant factor governing carrier injection, then similar $R_{C}$ values suggest that all systems may eventually yield equivalent performance, which is not the case; the data therefore suggests $\mathrm{R}_{\mathrm{C}}$ influences OECT operation at low $\mathrm{V}_{\mathrm{G}}$, but is unlikely to be the only factor governing the large increase in $\mathrm{g}_{\mathrm{m}}$ for Au:MBT:P-90 OECTs (Figure 2). 

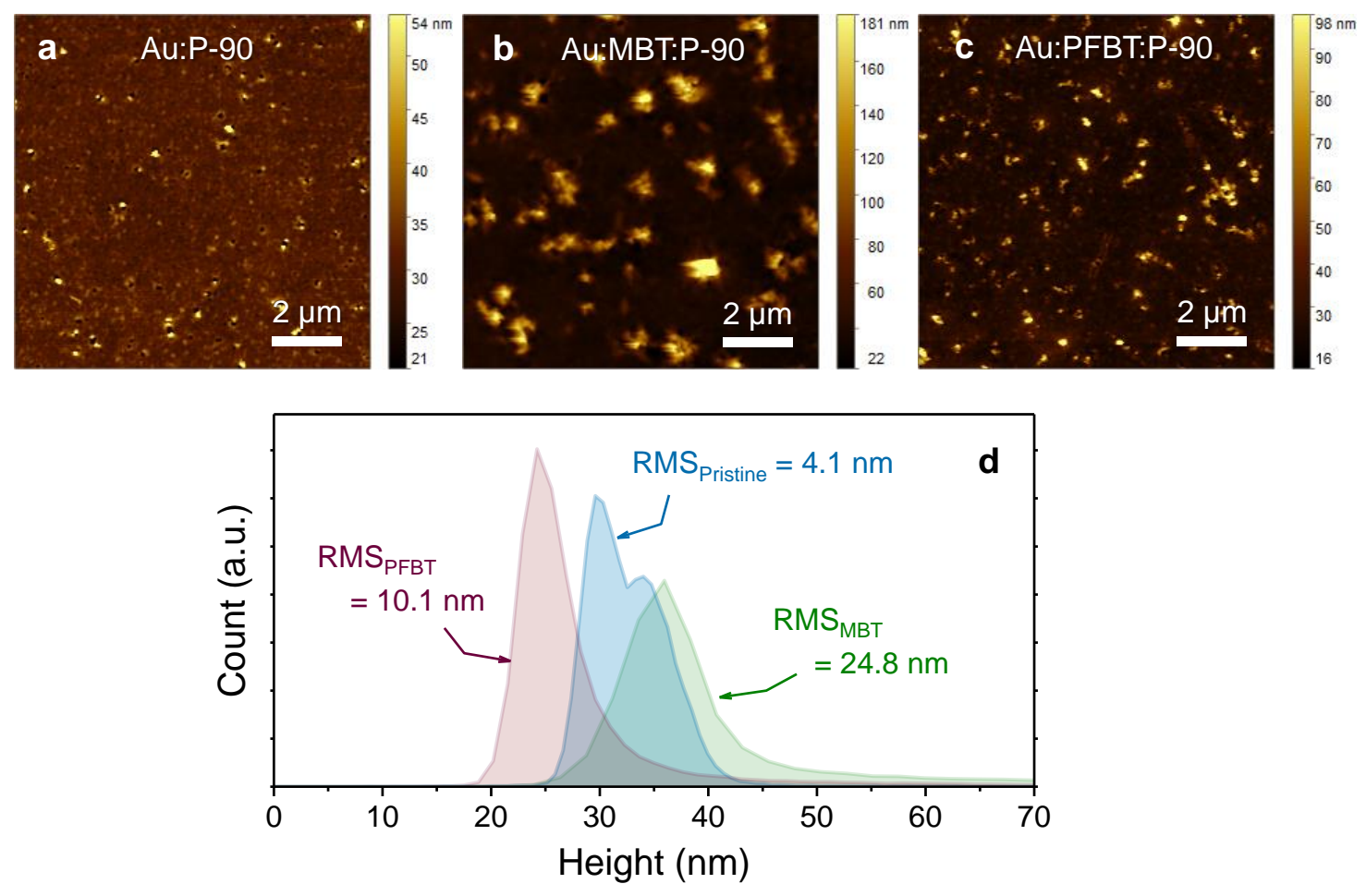

Figure 3. Atomic force microscopy (AFM) images showing the topography of P-90 thin-films formed on top of (a) gold, (b) and gold functionalised with MBT, and (c) gold functionalised with PFBT. Corresponding surface roughness and height distribution histograms are shown in (d). All films were deposited onto 580 x $580 \mu \mathrm{m}$ Au electrode-pads.

If $R_{C}$ alone cannot explain the stark differences in OECT performance, another possibility is SAM-induced surface energy modification of the Au electrodes, which can impact OSC wetting, solidification, and hence morphology. ${ }^{[39]}{ }^{[58] ~[43]}$ The latter can influence the OSC/metal interface and scale to the OSC in the transistor channel. We therefore used atomic force microscopy (AFM) to investigate the morphology of the OSC on the SAM-treated Au electrodes as well as the OECT channels. First, the surface topography AFM images (Figure 3a-c) reveal stark differences between Au:P-90, Au:PFBT:P-90 and Au:MBT:P-90 morphology at the electrodes. For instance, aggregates are present in all films, being smallest in Au:P-90 and significantly larger/more pronounced in films prepared on SAM-treated electrodes, particularly Au:MBT:P-90. We note 
that OSC morphology is also known to impact $\mathrm{RC}_{C}{ }^{[8]}$ which may explain the lower $\mathrm{RC}_{\mathrm{C}}$ in Au:PFBT:P-90 compared to Au:P-90. Further information at lower $\mathrm{V}_{\mathrm{G}}$, currently inhibited by poor TLM fits, would be required to clarify this trend.

Next, we studied the morphology in the OECT channels. Figure S6 shows further differences between morphology in Au:P-90, Au:PFBT:P-90 and Au:MBT:P-90 OECTs. In particular, the images and corresponding root mean square (RMS) of the surface roughness suggest improved thin-film quality/formation in the channel of the MBT-treated devices, which may enhance OECT operation. The surface roughness values at the channels are in contrast to those at

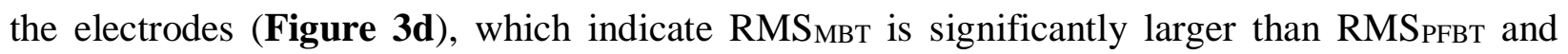
$\mathrm{RMS}_{\mathrm{Au}}$. The latter may indicate a larger surface area for ions to move through at the electrolyte/OSC interface, although it is likely that these values change during the OECT operation because of polymer swelling. ${ }^{[56]}{ }^{[57]}$ Although the exact morphology changes in the channel are different to those at the electrodes, the AFM images suggest that morphology differs between Au:P-90, Au:PFBT:P-90 and Au:MBT:P-90 OECT channels, and therefore morphology in the channel is influenced by SAM-treatment at the Au source/drain electrodes. These morphology differences can also influence mobility $(\mu)$ and volumetric capacitance $\left(\mathrm{C}^{*}\right),{ }^{[59]}$ the product of which $\left(\mu \times C^{*}\right)$ determines $g_{m}{ }^{[60]}{ }^{[61]}$ Table 1 summarises $\mu, C^{*}$ and $\mu \times C^{*}$, where $C^{*}$ is extracted from electrochemical impedance spectroscopy measurements (Figure S7), $\mu \times C^{*}$ is extracted from $\mathrm{ID}^{0.5}$ vs. $\mathrm{V}_{\mathrm{G}}$ (Figure S2) and $\mu$ is subsequently calculated. We find these critical parameters to be greatest in Au:MBT:P-90 devices. Although SAMs are used to prevent non-specific adsorption/binding in OECT sensing applications, ${ }^{[62]}$ up until now they have not been utilized to adjust metal/OSC interfacial properties; therefore our findings propose a new device engineering technique for improving morphology, $\mu, \mathrm{C}^{*}$ and $\mathrm{g}_{\mathrm{m}}$ in OECTs. 


\begin{tabular}{|c|c|c|c|c|c|c|c|c|c|}
\hline \multirow[b]{2}{*}{ Electrode } & \multicolumn{2}{|c|}{ Surface energy } & \multicolumn{6}{|c|}{ OECT properties } & \multirow{2}{*}{$\begin{array}{l}\mathbf{C V} \\
\mathrm{K}_{\mathrm{ET}} \\
\left(\mathrm{s}^{-1}\right)\end{array}$} \\
\hline & $\begin{array}{c}\gamma_{S}^{D} \\
(\mathrm{mN} / \mathrm{m})\end{array}$ & $\begin{array}{c}\gamma_{S}^{P} \\
(\mathrm{mN} / \mathrm{m})\end{array}$ & $\begin{array}{c}\mathrm{g}_{\mathrm{m} \_\max } \\
(\mu \mathrm{S})\end{array}$ & $\begin{array}{c}\mathrm{g}_{\mathrm{m} \_ \text {avg }} \\
(\mu \mathrm{S})\end{array}$ & $\begin{array}{c}\mu \times \mathrm{C}^{*} \\
\left(\mathrm{Fcm}^{-1} \mathrm{~V}^{-1} \mathrm{~s}^{-1}\right)\end{array}$ & $\begin{array}{c}\mathrm{C}^{*} \\
\left(\mathrm{Fem}^{-3}\right)\end{array}$ & $\begin{array}{c}\mu \\
\left(\mathrm{cm}^{2} / \mathrm{Vs}\right) \\
\end{array}$ & $\begin{array}{l}V_{\mathrm{T}} \\
(\mathrm{V})\end{array}$ & \\
\hline $\mathrm{Au}$ & 9.9 & 51.9 & 0.8 & 0.7 & 0.0009 & 91.6 & $9.8 \times 10^{-6}$ & 0.29 & 0.09 \\
\hline Au:MBT & 28.7 & 9.2 & 2.4 & 1.8 & 0.0023 & 132.5 & $1.7 \times 10^{-5}$ & 0.27 & 0.12 \\
\hline Au:PFBT & 19.7 & 14.1 & 0.5 & 0.5 & 0.0008 & 61.9 & $1.2 \times 10^{-5}$ & 0.29 & 0.06 \\
\hline
\end{tabular}

Table 1. A summary of the dispersive and polar surface energy components, $\gamma_{S}^{D}$ and $\gamma_{S}^{P}$, of the untreated gold electrodes and gold electrodes functionalized with PFBT and MBT, along with their

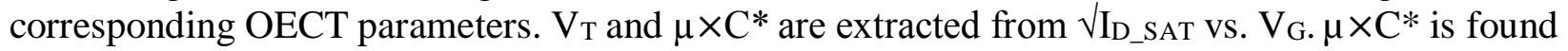
to be approximately three times greater in Au:MBT:P-90 devices than Au:P-90, when $\mathrm{V}_{\mathrm{T}}$ is taken into account, in accordance with ${ }^{[61]} . \mathrm{C}^{*}$ is determined using electrochemical impedance spectroscopy, and $\mu$ is calculated from $\mu \times \mathrm{C}^{*}$ and $\mathrm{C}^{*}$. $\mathrm{K}_{\mathrm{ET}}$ is the rate of electron transfer throughout the system.

Interestingly, the AFM images at the electrodes highlight numerous pinhole-like features (black circles) throughout the Au:P-90 film and twin-peaks in the Au:P-90 surface height histogram (Figure 3d). These features suggest the existence of two prominent heights/regimes, i.e. that the untreated Au surface has not been fully covered/wetted by the P-90. Therefore, to gain better insights into the surface properties of the SAM-treated Au electrodes, which subsequently impact OECT channel morphology, we investigated the relationship between the surface wetting of the various electrodes and OECT performance. ${ }^{[63][64]}$ The interactions between the electrode surfaces and the P-90 can be quantified using the Owens-Wendt-Kaelble method to calculate the polar and dispersive elements of the surface energy (see Experimental Section). ${ }^{[65]}$ Table 1 summarises the electrode surface energies, which were used to generate the wetting envelopes ${ }^{[63]}$ ${ }^{[64]}$ in Figure 4a at $\theta=0^{\circ}$. Given that a solution will only completely wet a surface if its surface tension components lie within the wetting envelope, Figure 4a shows that P-90 preferentially wets MBT-treated Au electrodes. This would improve the quality of the OSC/metal interface in Au:MBT:P-90 devices, underpinning the OSC morphology differences seen in the AFM images 
(Figure 3) and, in-turn, improve energetic disorder and electron injection/extraction. ${ }^{[66]}$ Furthermore, improved electrode wetting in the Au:MBT:P-90 devices likely corresponds to the improved thin-film formation in the channel (Figure S6), thereby contributing to overall improvement in MBT-treated OECTs. Additionally, the improved surface wetting may also impact OSC crystallinity. ${ }^{[67]}$ We therefore used X-ray diffraction (XRD) to investigate the Au:P-90, Au:MBT:P-90 and Au:PFBT:P-90 thin-films. Compared to Au:P-90, Figure 4b shows that peak intensity is increased in SAM-treated thin-films, indicating a higher degree of molecular ordering. Furthermore, the SAM-treated peaks are shifted towards lower angles, indicating increased dspacing and molecular orientation that is relatively more perpendicular with respect to the substrate. ${ }^{[68]}$ The overall SAM-induced improvements in surface wetting, morphology and crystallinity further demonstrate how influential S/D electrode treatments can be for OECTs.
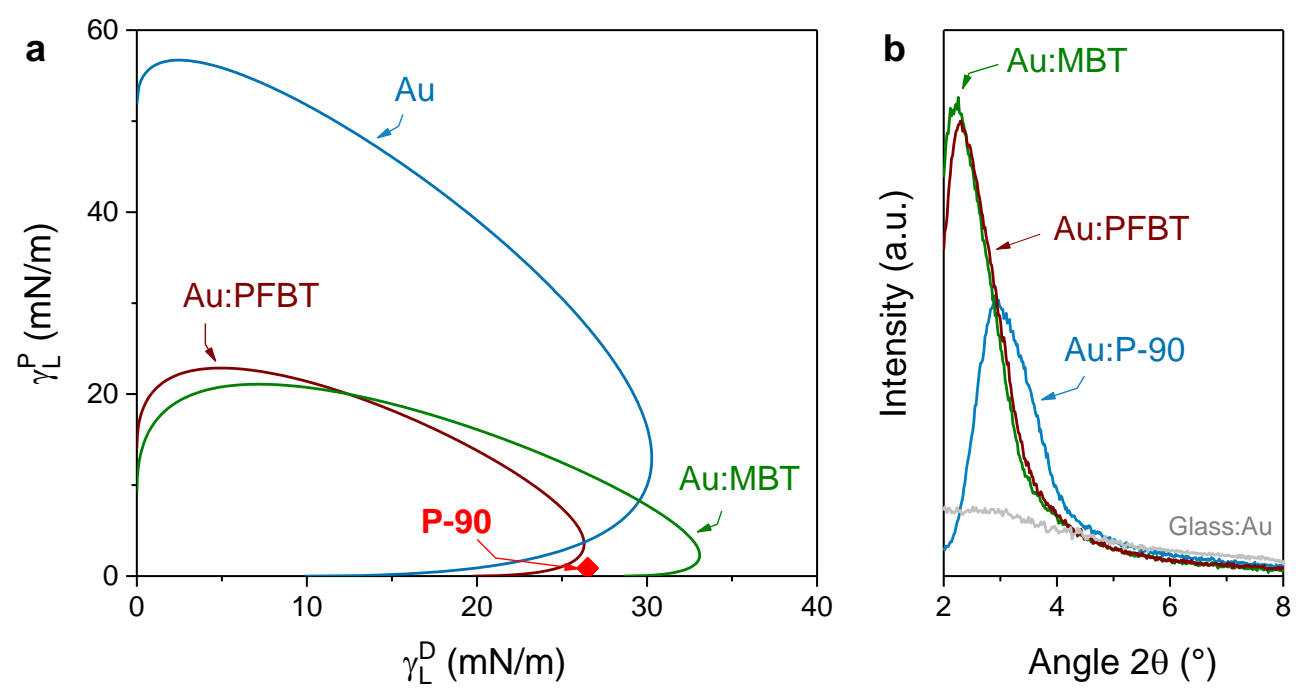

Figure 4. (a) Surface wetting envelope at a contact angle of $\theta=0^{\circ}$ for untreated gold electrodes and gold electrodes functionalised with PFBT and MBT. (b) X-ray diffraction measurements on Au:P-90, Au:MBT:P-90 and Au:PFBT:P-90 thin-films over a selected range of scanning angles $\left(2-8^{\circ}\right)$ to show features of interest. Corresponding measurements of pristine $\mathrm{Au}$ on the glass substrate (Glass:Au) are shown for comparison. 
Finally, Figure 4a shows that the wetting properties of P-90 are almost identical to chloroform (CF). These findings may therefore have broader implications, because CF-based solutions combined with Au-electrodes are popular in solution-processed bioelectronic systems due to their non-toxic, biocompatible and chemically inert properties. ${ }^{[69]}{ }^{[70]}$ In other words, modifying the surface energy of Au electrodes, or tailoring the solution-energy properties, can alter morphology in both the channel and at the electron injecting or extracting OSC/metal interface, improving the performance of numerous organic systems that are currently processed from CF. This further implies SAM treatments on S/D electrodes could have widespread impact in the field of OECTs.

In summary, whilst investigating the impact of contact resistance in n-type OECTs for the first time, we introduce a new device engineering approach for achieving state of the art n-type OECTs. We used the thiol-based SAMs, MBT and PFBT, to vary the potential barrier at the semiconductor/gold electrode interface, such that contact resistance is smallest in MBT-treated devices and greatest in PFBT-treated devices. We found that MBT-treatment increases n-type OECT transconductance to values greater than current state of the art. Further contact resistance analysis shows two gate voltage dependent operating regimes: (i) low gate voltage, where contact resistance influences OECT operation; (ii) high gate voltage, where ionic doping compensates for contact resistance. The latter contact resistance compensation suggests that contact resistance alone cannot explain the increase in transconductance. We therefore investigated the impact of the SAM treatments on semiconductor morphology in the channel and at the electrodes, revealing stark differences in morphology and crystallinity, and subsequent influences on mobility and capacitance. The collective results highlight SAM treatments on source/drain electrodes as new approach for improving OECT transconductance. To explore the reasons for the different 
morphologies we investigated the surface energy of the treated and untreated electrodes, finding that the semiconductor solution preferentially wets the MBT-treated gold electrodes, improving the quality of the semiconductor/electrode interface and subsequently improving semiconductor film-formation in the channel. On the other hand, organic semiconductor thin-films do not completely cover untreated gold, suggesting broader implications for this device engineering technique within the field of OECTs. Overall, we present the first analysis on contact resistance in n-type OECTs, whilst introducing a new and highly promising interfacial treatment technique for improving OECT performance.

\section{Experimental section}

Organic semiconductor and self-assembled monolayer preparation: P-90 was synthesized in-house and prepared in a $5 \mathrm{mg} \mathrm{ml}^{-1}$ chloroform solution. The MBT was stored in the glovebox and prepared in at a concentration of $1.8 \mathrm{mg} \mathrm{mL}^{-1}$ in acetonitrile. The gold electrodes were submerged in MBT-solution overnight in a dry nitrogen atmosphere, and annealed at $70^{\circ} \mathrm{C}$ for 10 minutes the next morning to remove any residual solvent. The PFBT was prepared at a ratio of 1:1000 in isopropyl alcohol. The gold electrodes were submerged in the PFBT-solution for 10 minutes and rinsed with isopropyl alcohol before drying with a nitrogen gun.

Electrochemical transistor fabrication and characterization: 4-inch glass wafers were cleaned by immersion in a piranha solution bath at $180^{\circ} \mathrm{C}$ for 10 minutes, subsequently immersed in a water bath and dried under a nitrogen flow. To ensure complete removal of contaminants, the wafers were exposed to an $\mathrm{O}_{2}$ plasma (Nanoplas DSB 6000). To define the geometry of the transistor, two photoresists were employed, forming a bilayer structure that facilitates lift-off after metal sputtering. This bilayer consisted of LOR 5B (Microchem) and S1813 (Shipley), individually spun 
and baked. Dissolution of the resist in the desired geometry was achieved by exposure to UV light (EVG 6200 mask alignment system) through a photomask and subsequent immersion in MF-319 developer. Metal contacts were defined by depositing a $10 \mathrm{~nm}$ Cr adhesion layer and a $100 \mathrm{~nm} \mathrm{Au}$ conductive layer (Equipment Support Company Ltd. ESCRD4) ensued by lift-off in organic solvents. The substrate surfaces were activated in an $\mathrm{O}_{2}$ plasma before vaporisation of a $1.7 \mu \mathrm{m}$ parylene C film (SCS Labcoater 2) which was adhered to the wafers using 3-(trimethoxysilyl)propyl methacrylate. For polymer patterning, a second parylene C layer, acting as a sacrificial layer, was vaporised. AZ9260 (Microchemicals) was utilised to define the channels, gates, and pads on the parylene $\mathrm{C}$ film, which were later etched using a reactive ion etcher (Oxford Instruments Plasmalab 100 - ICP 380). The Au source/drain electrodes were treated for 10 minutes with UV-ozone prior to functionalisation with MBT and PFBT, using the methods described above. The P-90 solution was then spin-coated onto the substrates at $1000 \mathrm{rpm}$ for 30 seconds. A Keithley 2606A SourceMeter unit was used to measure device transfer and output characteristics in a top-gate OECT configuration, with $\mathrm{Ag} / \mathrm{AgCl}$ as the gate electrode and $\mathrm{NaCl}$, at a concentration of $100 \mathrm{mM}$ in water, as the electrolyte. MATLAB and Origin were subsequently used to analyse the current-voltage data. Film thicknesses were measured on the lateral Au gate patterns $(580 \mu \mathrm{m}$ x $580 \mu \mathrm{m}$ ), coated at the same time and next to the active channels. A Dektak Profilometer was used to measure the film thicknesses $(\approx 135 \mathrm{~nm})$ in each system.

Ultraviolet photoelectron spectroscopy: UPS measurements were recorded using a SPECS PHOIBOS 100 hemispherical electron energy analyzer in a custom built ultrahigh vacuum (UHV) system with a base pressure of $\sim 1 \times 10^{-10}$ mbar. Samples were excited at $21.21 \mathrm{eV}$ using a $\mathrm{He} \mathrm{I}$ plasma source. 
Kelvin Probe: The work function differences between the untreated and SAM treated gold surfaces was determined via Kelvin Probe measurements inside a nitrogen environment. A KP Technology SPS040 system was used to acquire the data. To translate the relative differences to absolute values, a value of $-5.1 \mathrm{eV}$ for untreated gold was assumed. ${ }^{[24]}$

Electrochemical Impedance Spectroscopy: Electrochemical impedance spectroscopy (EIS) was performed using an Autolab PGSTAT128N potentiostat-galvanostat, from $10 \mathrm{kHz}$ to $0.1 \mathrm{~Hz}$, in a three-electrode setup. The measurements were performed at either a DC offset potential (doping) or at $\mathrm{V}_{\mathrm{OC}}$ with an $\mathrm{AC}$ amplitude of $10 \mathrm{mV}$. Capacitance-frequency data were plotted using the complex impedance formula: $Z=\sqrt{ }\left(R^{\wedge} 2+1 /\left(\omega^{\wedge} 2 C^{\wedge} 2\right)\right)$ The effective capacitance $(C)$ was then calculated using: $\mathrm{C} \sim 1 /(2 \pi f \ln (\mathrm{Z}))$, where $\mathrm{Z}$ is complex frequency component and $\mathrm{f}$ is frequency. Finally, $\mathrm{C}^{*}$ was extracted by normalizing the capacitance with the volume of the film.

Cyclic voltammetry measurements: CV data were obtained using an Autolab PGSTAT128N potentiostat-galvanostat. A three-electrode set-up was used, with, $\mathrm{Ag} / \mathrm{AgCl}, \mathrm{Pt}$ and $\mathrm{P}-90$ coated $\mathrm{Au}$ electrodes as the reference, counter and working electrodes, respectively. As per the OECT measurements, an aqueous $\mathrm{NaCl}$ solution $(100 \mathrm{mM})$ was used as the electrolyte. Data was acquired at a scan rate of $0.05 \mathrm{~V} \mathrm{~s}^{-1}$ and analysed using MATLAB and Metrohm NOVA.

Atomic force microscopy: AFM measurements were conducted in tapping mode using a Dimension Icon SPM. Gwyddion software was used for statistical analysis and data processing.

Surface energy/contact angle: The static contact angles of three liquids, namely water, ethylene glycol and benzyl alcohol, was measured on untreated as well as treated gold surfaces in order to determine their surface energies. Following the Owens-Wendt-Kaelble method, the total surface energy values as well as the breakdown into polar and dispersive components were determined, through which the wetting envelopes could be constructed. The instrument used in the process was 
a drop shape analyser DSA100 by Krüss. Additionally, the pendant drop method was used to evaluate the total surface tension of the P-90 polymer solution in CF. The polar $\left(\sigma_{P 90}^{p o l}\right)$ and dispersive $\left(\sigma_{P 90}^{\text {disp }}\right)$ surface tension components of the P-90 solution were determined by contact angle $(\theta)$ measurements on PTFE as a standard surface with measured surface energy $\left(\sigma_{P T F E}\right) .{ }^{[71]}$ First, the dispersive part was calculated according to:

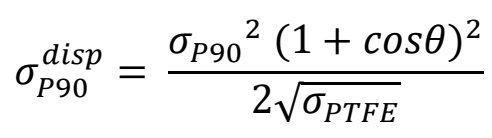

Then the polar part was found as the difference between the total surface tension and the dispersive part.

$$
\sigma_{P 90}^{p o l}=\sigma_{P 90}-\sigma_{P 90}^{d i s p}
$$

X-ray diffraction: X-ray diffraction measurements were performed with a Bruker D8 ADVANCE diffractometer at room temperature. The thin-films were studied in powder diffraction mode using Bragg-Brentano geometry ( $\theta-2 \theta$ scan configuration). A monochromatic $\mathrm{Cu} \mathrm{K} \alpha$ radiation beam with wavelength $\lambda=0.154 \mathrm{~nm}$ was used. The detector was scanned in the plane defined by the incidence beam and the surface normal. In this geometry, only polymer crystallites with a reciprocal lattice vector perpendicular to the film surface were detected.

\section{Acknowledgements}

S.I., A.F.P., H.F., A.S., G. N., M. G., T.C.H., X.C., I. M. and T.D.A. acknowledge King Abdullah University of Science and Technology (KAUST) for their financial support.

\section{References}


[1] A. D. Franklin, Science 2015, 349.

[2] H. L. Kwok, IEE Proceedings G - Circuits, Devices and Systems 1989, 136, 138.

[3] A. F. Paterson, T. D. Anthopoulos, Nature communications 2018, 9, 5264.

[4] Y. Xu, H. Sun, Y. Y. Noh, IEEE Trans. Electron Devices 2017, 64, 1932.

[5] C. Liu, Y. Xu, Y.-Y. Noh, Materials Today 2015, 18, 79.

[6] Y. Xu, H. Sun, W. Li, Y.-F. Lin, F. Balestra, G. Ghibaudo, Y.-Y. Noh, Adv. Mater. 2017, 29, 1702729.

[7] M. Marinkovic, D. Belaineh, V. Wagner, D. Knipp, Adv. Mater. 2012, 24, 4005.

[8] E. G. Bittle, H. W. Ro, C. R. Snyder, S. Engmann, R. J. Kline, X. R. Zhang, O. D. Jurchescu, D. M. DeLongchamp, D. J. Gundlach, J. Polym. Sci. Pt. B-Polym. Phys. 2017, 55, 1063.

[9] C. Liu, Y. Xu, Y. Li, W. Scheideler, T. Minari, The Journal of Physical Chemistry C 2013, 117, 12337.

[10] G. B. Blanchet, C. R. Fincher, M. Lefenfeld, J. A. Rogers, Appl. Phys. Lett. 2004, 84, 296.

[11] A. F. Paterson, A. D. Mottram, H. Faber, M. R. Niazi, Z. Fei, M. Heeney, T. D. Anthopoulos, Advanced Electronic Materials, 0, 1800723.

[12] R. Schroeder, L. A. Majewski, M. Grell, Applied Physics Letters 2004, 84, 1004.

[13] L. Bürgi, T. J. Richards, R. H. Friend, H. Sirringhaus, Journal of Applied Physics 2003, 94, 6129.

[14] Y. Takahashi, T. Hasegawa, Y. Abe, Y. Tokura, G. Saito, Applied Physics Letters 2006, 88, 073504.

[15] A. F. Paterson, S. Singh, K. J. Fallon, T. Hodsden, Y. Han, B. C. Schroeder, H. Bronstein, M. Heeney, I. McCulloch, T. D. Anthopoulos, Advanced Materials 2018, 30, 1801079.

[16] D. Natali, M. Caironi, Adv. Mater. 2012, 24, 1357.

[17] H. H. Choi, K. Cho, C. D. Frisbie, H. Sirringhaus, V. Podzorov, Nature Materials 2017, 17, 2.

[18] M. Ghittorelli, L. Lingstedt, P. Romele, N. I. Crăciun, Z. M. Kovács-Vajna, P. W. M. Blom, F. Torricelli, Nature Communications 2018, 9, 1441.

[19] J. Rivnay, S. Inal, A. Salleo, R. M. Owens, M. Berggren, G. G. Malliaras, Nature Reviews Materials 2018, 3, 17086.

[20] P. Gkoupidenis, D. A. Koutsouras, G. G. Malliaras, Nature Communications 2017, 8, 15448.

[21] J. Y. Gerasimov, R. Gabrielsson, R. Forchheimer, E. Stavrinidou, D. T. Simon, M. Berggren, S. Fabiano, Advanced Science 2019, 6, 1801339.

[22] D. Khodagholy, T. Doublet, P. Quilichini, M. Gurfinkel, P. Leleux, A. Ghestem, E. Ismailova, T. Hervé, S. Sanaur, C. Bernard, G. G. Malliaras, Nature Communications 2013, 4, 1575.

[23] D. Khodagholy, J. Rivnay, M. Sessolo, M. Gurfinkel, P. Leleux, L. H. Jimison, E. Stavrinidou, T. Herve, S. Sanaur, R. M. Owens, G. G. Malliaras, Nature Communications 2013, 4, 2133.

[24] W. M. H. Sachtler, G. J. H. Dorgelo, A. A. Holscher, Surface Science 1966, 5, 221.

[25] M. Moser, J. F. Ponder Jr., A. Wadsworth, A. Giovannitti, I. McCulloch, Advanced Functional Materials, 0, 1807033.

[26] H. Sun, J. Y. Gerasimov, M. Berggren, S. Fabiano, Journal of Materials Chemistry C 2018. 
[27] A. Giovannitti, C. B. Nielsen, D.-T. Sbircea, S. Inal, M. Donahue, M. R. Niazi, D. A. Hanifi, A. Amassian, G. G. Malliaras, J. Rivnay, I. McCulloch, Nature Communications 2016, 7, 13066.

[28] V. Kaphle, S. Liu, A. Al-Shadeedi, C.-M. Keum, B. Lüssem, Advanced Materials 2016, 28, 8766.

[29] J. T. Friedlein, J. Rivnay, D. H. Dunlap, I. McCulloch, S. E. Shaheen, R. R. McLeod, G. G. Malliaras, Applied Physics Letters 2017, 111, 023301.

[30] H. Sun, M. Vagin, S. Wang, X. Crispin, R. Forchheimer, M. Berggren, S. Fabiano, Advanced Materials 2018, 30, 1704916.

[31] A. M. Pappa, D. Ohayon, A. Giovannitti, I. P. Maria, A. Savva, I. Uguz, J. Rivnay, I. McCulloch, R. M. Owens, S. Inal, Science Advances 2018, 4.

[32] Y. Xue, X. Li, H. Li, W. Zhang, Nature Communications 2014, 5, 4348.

[33] Z. A. Lamport, K. J. Barth, H. Lee, E. Gann, S. Engmann, H. Chen, M. Guthold, I. McCulloch, J. E. Anthony, L. J. Richter, D. M. DeLongchamp, O. D. Jurchescu, Nature communications 2018, 9, 5130 .

[34] G. Witte, S. Lukas, P. S. Bagus, C. Wöll, Applied Physics Letters 2005, 87, 263502.

[35] Y. H. Zhou, C. Fuentes-Hernandez, J. Shim, J. Meyer, A. J. Giordano, H. Li, P. Winget, T. Papadopoulos, H. Cheun, J. Kim, M. Fenoll, A. Dindar, W. Haske, E. Najafabadi, T. M. Khan, H. Sojoudi, S. Barlow, S. Graham, J. L. Bredas, S. R. Marder, A. Kahn, B. Kippelen, Science 2012, 336, 327.

[36] N. Cernetic, O. Acton, T. Weidner, D. O. Hutchins, J. E. Baio, H. Ma, A. K. Y. Jen, Organic Electronics 2012, 13, 3226.

[37] B. Fu, C.-Y. Wang, B. D. Rose, Y. Jiang, M. Chang, P.-H. Chu, Z. Yuan, C. Fuentes-Hernandez, B. Kippelen, J.-L. Brédas, D. M. Collard, E. Reichmanis, Chemistry of Materials 2015, 27, 2928.

[38] Y. Kuzumoto, M. Kitamura, Applied Physics Express 2014, 7, 035701.

[39] K. Asadi, F. Gholamrezaie, E. C. P. Smits, P. W. M. Blom, B. d. Boer, Journal of Materials Chemistry 2007, 17, 1947.

[40] G. Heimel, L. Romaner, J.-L. Brédas, E. Zojer, Physical Review Letters 2006, 96, 196806.

[41] G. Heimel, L. Romaner, E. Zojer, J.-L. Bredas, Accounts of Chemical Research 2008, 41, 721.

[42] F. Rissner, G. M. Rangger, O. T. Hofmann, A. M. Track, G. Heimel, E. Zojer, ACS Nano 2009, 3, 3513.

[43] J. Semple, D. G. Georgiadou, G. Wyatt-Moon, M. Yoon, A. Seitkhan, E. Yengel, S. Rossbauer, F. Bottacchi, M. A. McLachlan, D. D. C. Bradley, T. D. Anthopoulos, npj Flexible Electronics 2018, 2,18 .

[44] K. Yasutaka, K. Masatoshi, Applied Physics Express 2014, 7, 035701.

[45] T. Minari, T. Miyadera, K. Tsukagoshi, Y. Aoyagi, H. Ito, Appl. Phys. Lett. 2007, 91, 053508.

[46] E. Meijer, G. Gelinck, E. Van Veenendaal, B.-H. Huisman, D. De Leeuw, T. Klapwijk, Applied physics letters 2003, 82, 4576.

[47] A. F. Paterson, Y.-H. Lin, A. D. Mottram, Z. Fei, M. R. Niazi, A. R. Kirmani, A. Amassian, O. Solomeshch, N. Tessler, M. Heeney, T. D. Anthopoulos, Advanced Electronic Materials, 1700464.

[48] T. Uemura, C. Rolin, T.-H. Ke, P. Fesenko, J. Genoe, P. Heremans, J. Takeya, Adv. Mater. 2016, $28,151$. 
[49] C. Liu, G. Li, R. Di Pietro, J. Huang, Y.-Y. Noh, X. Liu, T. Minari, Phys. Rev. Appl. 2017, 8, 034020 .

[50] D. Pletcher, R. Greff, R. Peat, L. Peter, J. Robinson, Instrumental methods in electrochemistry, Elsevier, 2001.

[51] R. J. Klingler, J. K. Kochi, The Journal of Physical Chemistry 1981, 85, 1731.

[52] A. J. Bard, L. R. Faulkner, J. Leddy, C. G. Zoski, Electrochemical methods: fundamentals and applications, Vol. 2, wiley New York, 1980.

[53] B. Lüssem, M. Riede, K. Leo, physica status solidi (a) 2013, 210, 9.

[54] J. Liu, X. Liu, Z. Chen, L. Miao, X. Liu, B. Li, L. Tang, K. Chen, Y. Liu, J. Li, Z. Wei, X. Duan, Nano Research 2019, 12, 463.

[55] Y. Xu, H. Sun, A. Liu, H.-H. Zhu, W. Li, Y.-F. Lin, Y.-Y. Noh, Advanced Materials 2018, 30, 1801830.

[56] A. Savva, C. Cendra, A. Giugni, B. Torre, J. Surgailis, D. Ohayon, A. Giovannitti, I. McCulloch, E. Di Fabrizio, A. Salleo, J. Rivnay, S. Inal, Chemistry of Materials 2019.

[57] C. Cendra, A. Giovannitti, A. Savva, V. Venkatraman, I. McCulloch, A. Salleo, S. Inal, J. Rivnay, Advanced Functional Materials 2019, 29, 1807034.

[58] M. Sarcletti, T. Schmaltz, M. Halik, Organic Electronics 2014, 15, 3082.

[59] S. Inal, J. Rivnay, P. Leleux, M. Ferro, M. Ramuz, J. C. Brendel, M. M. Schmidt, M. Thelakkat, G. G. Malliaras, Advanced Materials 2014, 26, 7450.

[60] J. Rivnay, P. Leleux, M. Ferro, M. Sessolo, A. Williamson, D. A. Koutsouras, D. Khodagholy, M. Ramuz, X. Strakosas, R. M. Owens, C. Benar, J.-M. Badier, C. Bernard, G. G. Malliaras, Science Advances 2015, 1, e1400251.

[61] S. Inal, G. G. Malliaras, J. Rivnay, Nature Communications 2017, 8, 1767.

[62] D. Gentili, P. D’Angelo, F. Militano, R. Mazzei, T. Poerio, M. Brucale, G. Tarabella, S. Bonetti, S. L. Marasso, M. Cocuzza, L. Giorno, S. Iannotta, M. Cavallini, Journal of Materials Chemistry B 2018, 6, 5400.

[63] J. M. Ball, P. H. Wöbkenberg, F. Colléaux, M. Heeney, J. E. Anthony, I. McCulloch, D. D. C. Bradley, T. D. Anthopoulos, Applied Physics Letters 2009, 95, 103310.

[64] P. H. Wöbkenberg, J. Ball, F. B. Kooistra, J. C. Hummelen, D. M. d. Leeuw, D. D. C. Bradley, T. D. Anthopoulos, Applied Physics Letters 2008, 93, 013303.

[65] D. Y. Kwok, A. W. Neumann, Advances in Colloid and Interface Science 1999, 81, 167.

[66] H. Sirringhaus, Advanced Materials 2014, 26, 1319.

[67] S. Riera-Galindo, A. Tamayo, M. Mas-Torrent, ACS Omega 2018, 3, 2329.

[68] H.-W. Zan, C.-W. Chou, Japanese Journal of Applied Physics 2009, 48, 031501.

[69] Y. Khan, F. J. Pavinatto, M. C. Lin, A. Liao, S. L. Swisher, K. Mann, V. Subramanian, M. M. Maharbiz, A. C. Arias, Advanced Functional Materials 2016, 26, 1004.

[70] B. Cornell, V. Krishnamurthy, W. Hoiles, in Dynamics of Engineered Artificial Membranes and Biosensors, Cambridge University Press, Cambridge 2018, i.

[71] W. D. Kaplan, D. Chatain, P. Wynblatt, W. C. Carter, Journal of Materials Science 2013, 48, 5681. 